\title{
Estrategias y metodologias didácticas, una mirada desde su aplicación en los programas de Administración
}

\begin{tabular}{|l|}
\hline Wilson Montenegro-Velandia \\
\hline Universidad Cooperativa de Colombia, \\
Colombia \\
wilson.montenegro@campusucc.edu.co
\end{tabular}

Iván Darío Toro-Jaramillo

Universidad de Medellín, Colombia idtoro@udem.edu.co

Cesar Alveiro Montoya-Agudelo

Universidad Cooperativa de Colombia, Colombia

cesar.montoyaag@campusucc.edu.co

\section{Pastor Emilio Pérez-Villa}

Universidad Cooperativa de Colombia, Colombia

pastor.perez@campusucc.edu.co

\section{Ana María Cano-Arroyave}

Institución Universitaria Esumer, Colombia

ana.cano@esumer.edu.co

\section{Jhon Jaime Arango-Benjumea}

Universidad Cooperativa de Colombia, Colombia

jhon.arangob@campusucc.edu.co

Juan Esteban Vahos-Correa
Universidad Cooperativa de Colombia,
Colombia
jevahosc@escolme.edu.co

Bladimir Coronado-Ríos

Universidad Cooperativa de Colombia, Colombia

bladimir.coronado@campusucc.edu.co

\section{Resumen}

El propósito del artículo es caracterizar la didáctica utilizada en programas universitarios de Administración. La investigación se llevó a cabo en once universidades ubicadas en Medellín (Colombia). La metodología aplicada es de tipo mixto; cuenta con un diseño exploratorio y descriptivo. La información se recogió con una muestra de 206 estudiantes, tres grupos focales con docentes, cada uno con seis personas, entrevistas con directivos de programas y análisis de proyectos educativos. Entre los hallazgos, se encontró que hay pocas relaciones entre las estrategias didácticas y las operaciones de pensamiento desarrolladas por los estudiantes, una de las posibles causas es la fuerte preferencia de los profesores por la transmisión de contenidos, aunque es posible identificar una tendencia a utilizar estrategias didácticas basada en competencias. Se concluye que en los programas de Administración, las metodologías didácticas se enfocan cada vez más en la formación por competencias de los estudiantes. Sin embargo, esta tendencia sigue siendo tímida y, en algunos casos, desalineada con los objetivos de formación.

\section{Palabras clave}

Desarrollo de competencias; método de enseñanza; pedagogía universitaria; técnica didáctica; enseñanza de la Administración (Fuente: Tesauro de la Unesco).

Recepción: 11/12/2015 | Envío a pares: 15/02/2016 | Aceptación por pares: 11/04/2016 | Aprobación: 17/04/2016

Montenegro-Velandia, W., Cano-Arroyave, A., Toro-Jaramillo, I., Arango-Benjumea, J., Montoya-Agudelo, C., Vahos-Correa, J., PérezVilla, P., y Coronado-Ríos, B. (2016). Estrategias y metodologías didácticas, una mirada desde su aplicación en los programas de Administración. Educ. Educ., 19(2), 205-220. DOI: 10.5294/edu.2016.19.2.2 


\title{
Teaching Strategies: A Look at their Application in Business Administration Programs
}

\begin{abstract}
The purpose of this article is to characterize the didactics used in college business administration programs. The research was conducted at eleven universities in the city of Medellin (Colombia). A mixed methodology was used, based on an exploratory and descriptive design. The information was collected from a sample of 206 students; threefocus groups with teachers, each including six participants; interviews with program directors; and an analysis of educational projects. Among the findings, it was discovered there is little relationship between teaching strategies and the thought processes developed by students. One of the possible causes is the clear-cut preference among teachers for transmitting content, although it is possible to identify a tendency to use skill-based teaching strategies. The conclusion is that the teaching methods in business administration programs focus increasingly on helping students to develop skills. Nevertheless, this trend is still timid and, in some cases, out of line with the educational objectives of these programs.
\end{abstract}

\section{Keywords}

Development of skills; teaching method; university education; didactic technique; teaching business administration (Source: Unesco Thesaurus) 


\section{Estratégias didáticas: um olhar a partir de sua aplicação utilizada nos programas de administração}

Resumo

O propósito deste artigo é caracterizar a didática utilizada em programas universitários de administração. A pesquisa foi realizada em 11 universidades de Medellín (Colômbia). A metodologia aplicada é de tipo misto; conta com um desenho exploratório e descritivo. A informação foi coletada com uma amostra de 206 estudantes, três grupos focais com docentes, cada um com seis pessoas, entrevistas com diretores de programas e análises de projetos educativos. Entre os achados, constatou-se que há poucas relações entre as estratégias didáticas e as operações de pensamento desenvolvidas pelos estudantes, uma das possiveis causas é a forte preferência dos professores pela transmissão de conteúdos, embora seja possivel identificar uma tendência a utilizar estratégias didáticas baseada em competências dos estudantes. Contudo, essa tendência continua sendo tímida e, em alguns casos, desalinhada com os objetivos de formação.

\section{Palavras-chave}

Desenvolvimento de competências, método de ensino, pedagogia universitária, técnica didática, ensino da administração (Fonte: Tesauro da Unesco). 


\section{Introducción}

La enseñanza y el aprendizaje de la administración requieren la utilización de una didáctica que lleve efectivamente a la formación de los estudiantes. La didáctica de la administración está sometida a exigencias relacionadas con la pertinencia, calidad y cobertura, de manera que pueda responder a las dinámicas actuales de la formación del administrador, las cuales promueven el pensamiento crítico y sistémico, las habilidades en la toma de decisiones, en el análisis del contexto y en el direccionamiento estratégico de las organizaciones (Brown, Bimrose, Barnes y Hughes, 2012; Fung, 2014; Lim y Lee, 2014; Pedraza et al., 2013). En el campo de la administración, la principal preocupación ha sido el pensamiento disciplinar, dejando de lado la reflexión sobre su didáctica (Toro et al., 2013). En este sentido, cobra importancia el análisis de la didáctica en los programas de esta disciplina. El propósito de este estudio es describir las metodologías didácticas que utilizan los profesores, con el fin de aportar elementos que contribuyan a la comprensión y el mejoramiento de este aspecto en la administración.

\section{Marco teórico}

\section{Elementos de la didáctica}

Al hacer el análisis de los diferentes conceptos que se tienen de didáctica, se encontró que lo común es la teorización en torno al proceso de enseñanzaaprendizaje. La didáctica articula la teoría y la práctica en este el proceso; Lucio (1989) la define como "el saber que tematiza el proceso de instrucción"; De Jesús, Méndez, Andrade y Martínez (2007) afirman que "la didáctica es un saber reflexionado y tematizado, una teoría sobre la enseñanza que indica caminos y horizontes promisorios para la formación" (p. 12). En este proceso reflexivo es necesario precisar que la didáctica comprende estrategias, modalidades y metodologías para lograr el desarrollo formativo.

En primer lugar, la estrategia didáctica es entendida como la planificación de la forma como se llevará a cabo el proceso formativo y comprende un diagnóstico de la realidad, la definición de objetivos, la selección de métodos didácticos, las tareas por realizar, las actividades, la planeación de los recursos y la forma de evaluación del proceso formativo (Burgess y Russell, 2003; Cid-Sabucedo, Pérez-Abellás y Zabalza, 2009b; González y Ramírez, 2010).

En segundo lugar, las modalidades de enseñanza se entienden como los distintos escenarios donde tienen lugar las actividades definidas en la estrategia didáctica. Estas modalidades se clasifican como actividades presenciales y no presenciales (De Miguel et al., 2005). En las primeras hay una intervención directa de profesores y estudiantes, mientras que en las segundas los estudiantes realizan actividades de forma individual o grupal.

En tercer lugar, la metodología didáctica se entiende como la acción sistematizada de seleccionar y organizar las actividades, los recursos y los tiempos para alcanzar los objetivos de formación definidos por la estrategia didáctica. En este sentido, Villamizar-Herrera, Montenegro-Velandia y Salvador-Poveda (2012) la definen como "un proceso intencionado de apropiación del conocimiento que se inicia con la reflexión, comprensión, construcción y evaluación de las acciones didácticas que propician la adquisición y el desarrollo de habilidades y actitudes para un adecuado desempeño en la sociedad" (pp. 277-278). Por tanto, la metodología didáctica es la que propicia en forma directa del proceso de enseñanza-aprendizaje. De esta manera, mientras la estrategia didáctica define lo que se va a hacer y la metodología didáctica define el cómo, la modalidad de enseñanza define el escenario.

\section{Metodologías didácticas}

En el análisis de la literatura se encontraron diferentes formas de clasificar las metodologías didácticas. Amat (1998) y Nérici (1985) las clasifican a partir de las formas de razonamiento (inductivo, deductivo, analógico), la actividad de los estudiantes (pasivo, activo) y el trabajo de los estudiantes (individual, colectivo), mientras que Verona (2004) hace 
una clasificación de acuerdo con la modalidad de enseñanza. Para este estudio se tomó como guía el trabajo presentado por De Miguel et al. (2005), quienes hacen una clasificación a partir de la individualidad, la socialización y la interdisciplinariedad, la cual está relacionada con la forma de participación del estudiante en su proceso de aprendizaje.

En este orden de ideas, el enfoque individual utiliza como metodologías la tutoría académica, el seminario alemán, el aula virtual o la enseñanza programada y la investigación formativa. La tutoría académica es un acompañamiento del profesor al estudiante y un apoyo a otras metodologías. El seminario alemán es una forma de investigación en la que los estudiantes entran en contacto con los marcos teóricos existentes y a través del debate van construyendo su conocimiento. El aula virtual o enseñanza programada se basa en el uso de las nuevas tecnologías de la información y la comunicación (TIC), donde los tiempos y los lugares de estudio no son necesariamente los mismos. La investigación formativa incluye la identificación y definición de un problema, la forma de abordarlo, la búsqueda y sistematización de la información, la interpretación de los resultados y la presentación de conclusiones, lo cual trasciende el desarrollo de temas abordando los problemas desde la interdisciplinariedad (De Miguel et al., 2005; Pedraza et al., 2013; Verona, 2004). En términos generales, el enfoque individual centra su atención en el estudiante como sujeto.

En cuanto a los métodos de socialización didáctica, se subdivide en dos grupos de metodologías; en el primero hay una fuerte participación del profesor y en el segundo son los estudiantes los protagonistas del desarrollo del proceso didáctico. Dentro del primer grupo se encuentra la clase magistral, la cual consiste en la exposición verbal por parte del profesor y donde la participación o actividad del estudiante se reduce a escuchar. El método de caso se fundamenta en el análisis de un hecho, problema o suceso real bajo la guía del profesor a partir de una nota de enseñanza. La clase práctica es un complemento de la clase magistral, consiste en que los estudiantes apliquen las teorías y los métodos para resolver ejercicios o problemas propuestos. En el seminario o conferencia, los estudiantes se integran activamente en la discusión de un tema específico previamente consultado con la moderación del profesor (Herrero, 1996; Popil, 2011; Swanson et al., 2011). En estos métodos, el aspecto común es el papel protagónico del profesor como guía y acompañante del proceso de enseñanza-aprendizaje.

En el segundo grupo de metodologías de socialización, el profesor sigue acompañando el proceso con una menor participación. Entre estas, la tutoría entre estudiantes, de acuerdo con Alzate-Medina y Peña-Borrero (2010): "Es una modalidad de aprendizaje cooperativo, en la que estudiantes más aventajados apoyan el aprendizaje de otros menos expertos, mediante un trabajo que se realiza en grupos pequeños o en parejas" (p. 125). Otro método es el de grupos heterogéneos de aprendizaje cooperativo, según las aptitudes y el grado de experticia de los participantes; la tutoría puede darse entre estudiantes de edades o cursos diferentes con el fin de abordar problemas propuestos desde distintas perspectivas. Por último, los grupos por centro de interés se conforman libremente en torno a los contenidos de aprendizaje que consideran más interesantes y están motivados por la intención de comprender (Baeten, Kyndt, Struyven y Dochy, 2010; Cid-Sabucedo, Pérez-Abellás y Zabalza, 2009a; De Miguel et al., 2005; Fung, 2014; Tarabay, 2009). El rol del profesor en estas metodologías es el de orientador, además es quien propone las temáticas o problemáticas que se van a abordar.

Finalmente, las metodologías desde el enfoque de la globalización corresponden a aquellas que pueden abordar interdisciplinarmente la realidad, tales como el aprendizaje basado en problemas, la enseñanza basada en proyectos, los juegos de empresa o simulaciones y las visitas o prácticas empresariales. En cuanto al aprendizaje basado en problemas, se busca que el estudiante entienda una 
situación de la realidad, a partir de la identificación de sus propias necesidades, lo cual lo lleva a integrar conceptos y procedimientos, necesarios para la solución de un problema planteado con anterioridad (Lile y Kelemen, 2014; Morales y Landa, 2004). La enseñanza basada en proyectos es un método complementario con otros, debe entenderse como un proceso interactivo entre el aprendizaje, el mundo laboral, el individuo y el grupo, lo cual permite desarrollar una acción completa a través del análisis de las teorías, la planificación, la toma de decisiones, la ejecución, el control y la evaluación (Tippelt y Lindemann, 2007).

De igual forma, los juegos de empresa o simulaciones facilitan la experiencia interactiva asignando roles a cada miembro del equipo participante y promueven la práctica en un ambiente controlado; con mucha frecuencia, destaca aspectos cuantitativos de la toma de decisiones y requiere el uso de tecnologías de información y comunicación (Swanson et al., 2011). Las visitas o prácticas empresariales permiten tener un contacto con la problemática de las empresas y sus métodos de trabajo, facilitando la comprensión del trabajo desarrollado en el aula (De Miguel et al., 2005; Nikolova, van Ruysseveldt, De Witte y Syroit, 2014). Uno de los aspectos relevantes para destacar de estas metodologías es su aporte al aprendizaje por descubrimiento.

En el marco de las observaciones previas, Grisales-Franco y González-Agudelo (2009) afirman que "la práctica del profesor, implica no solo un conocimiento científico sobre un saber específico, sino también un conocimiento en didáctica" (p. 80), específicamente en metodologías didácticas. Del mismo modo, según Tarabay (2009, p. 361), son dos facetas por tener en cuenta: "Los conocimientos y las habilidades pedagógico-comunicativas. Es decir, lo que sabe y cómo transmite aquello que sabe". En este sentido, la presente investigación aborda las siguientes preguntas: ¿qué caracteriza las prácticas pedagógicas llevadas a cabo en las facultades de Administración en Colombia?, ¿qué pertinencia tie- nen las prácticas pedagógicas llevadas a cabo en las facultades de Administración para la formación del administrador actual?

Como consecuencia de todo presentado, se espera que el estímulo y el apoyo del profesor influyan en el desarrollo de las operaciones de pensamiento, las habilidades y el aprendizaje de los estudiantes.

\section{Metodología}

En un estudio previo se abordó la situación de la investigación en los programas de Administración, arrojando como resultado que la mayor preocupación de nuestras facultades y programas no estaba solo en el asunto de la investigación y los grupos de investigación, sino principalmente en la formación de los docentes en campos como la educación y la didáctica (Calderón et al., 2014). A partir de este trabajo surgió la actual investigación, en la que participaron once universidades de la Asociación Colombiana de Facultades de Administración (Ascolfa), con el propósito de hacer un aporte a la educación y a la didáctica en relación con el saber administrativo.

La actual investigación presenta un enfoque mixto de corte transversal, implicó un proceso de recolección y análisis de datos tanto cuantitativos como cualitativos. El estudio se desarrolló en cuatro fases o momentos: en la primera se hicieron encuestas a estudiantes de los programas de Administración, esta se llevó a cabo en forma virtual con un formulario de Google Docs; participaron 206 estudiantes de once instituciones de educación superior. La encuesta se respondió con seis opciones de escala Likert, las posibles elecciones de respuesta fueron: $1=$ no responde, 2 = nunca, $3=$ casi nunca, 4 = en ocasiones, $5=$ con frecuencia, 6 = siempre. La confiabilidad de la encuesta tuvo un alpha de Cronbach $=0,78$, lo cual representa una fuerte consistencia interna.

La segunda fase correspondió a grupos focales con profesores, cada uno con seis docentes. En la tercera fase se realizaron entrevistas a jefes de programa o decanos. De las fases dos y tres, se recogió informa- 
ción en audio y vídeo, la cual se transcribió, clasificó y analizó utilizando Atlas-ti. El análisis se hizo tomando las estrategias y las metodologías didácticas como las categorías principales. Sin embargo, al desarrollar este proceso surgieron otras categorías como: conocimientos, competencias, evaluación, medios y recursos. Finalmente, la fase cuatro consistió en el análisis de los Proyectos Educativos de los programas de Administración de las universidades participantes.

\section{Resultados y discusión}

\section{Hallazgos obtenidos en la encuesta a los estudiantes de los programas de Administración}

En la tabla 1 se destacan las operaciones de pensamiento que mayormente se promueven desde las estrategias didácticas que proponen los profesores. Se encontró que la confiabilidad compuesta de la mayoría de los constructos fue mayor de o,6o.

De acuerdo con Chesnut y Burley (2015), uno de los factores predictores de rendimiento de los estudiantes es la capacidad de los profesores. Sin embargo, como se observa en la tabla 1, se encuentran bajas relaciones entre las estrategias didácticas y las operaciones de pensamiento desarrolladas en los estudiantes. Esto puede ser debido a que las actividades diseñadas por los profesores no están pensadas con la intención de reforzar una operación de pensamiento o una competencia específica, sino en la presentación de un tema.

Adicionalmente, para Bailey y Card "el maestro que quiere convertirse en un facilitador del aprendizaje requiere un conjunto diferente de habilidades pedagógicas que se centran en ayudar a los estudiantes con el fin de desarrollar la comprensión de los contenidos" (2009, p. 153). En tal sentido, el conocimiento y la aplicación adecuada de metodologías didácticas por parte del profesor facilita en los estudiantes el desarrollo de operaciones de pensamiento. En la tabla 2 se presentan las relaciones porcentuales entre estas.

Se destaca el pensamiento reflexivo y la argumentación oral y escrita como las operaciones de pensamiento que mayormente se promueven con la aplicación de los estudios de caso, el seminario alemán, la investigación en el aula y los semilleros de investigación. Específicamente, se percibe que el seminario alemán influye en un $49 \%$ en el desarrollo del pensamiento reflexivo, la investigación en el aula con un $42 \%$ y un $32 \%$ en la argumentación oral y escrita respectivamente.

Tabla 1

Correlación entre las operaciones de pensamiento y los objetivos de las actividades por desarrollar

\begin{tabular}{|l|c|c|c|c|c|c|c|}
\hline & 1 & 2 & 3 & 4 & 5 & 6 & 7 \\
\hline 1. Memorización & 1 & & & & & & \\
\hline 2. Análisis & $0,247^{* *}$ & 1 & & & & & \\
\hline 4. Interpretación & $0,165^{*}$ & $0,516^{* *}$ & 1 & & & & \\
\hline 5. Argumentación & $0,163^{*}$ & $0,280^{* *}$ & $0,180^{* *}$ & 1 & & & \\
\hline 6. Pensamiento reflexivo & $0,184^{* *}$ &, $473^{* *}$ &, $409^{* *}$ &, $286^{* *}$ & 1 & & \\
\hline 7. Objetivos de las actividades por desarrollar & 0,105 & $0,143^{*}$ & $-0,005$ & $0,175^{*}$ & $0,190^{* *}$ & 1 & \\
\hline Consistencia interna & 0,060 & $0,148^{*}$ & 0,040 & $0,158^{*}$ & $0,186^{* *}$ & $0,368^{* *}$ & 1 \\
\hline
\end{tabular}

${ }^{* *} p<0,01$

${ }^{*} p<0,05$ 
Tabla 2

Relación entre metodologías didácticas y operaciones de pensamiento

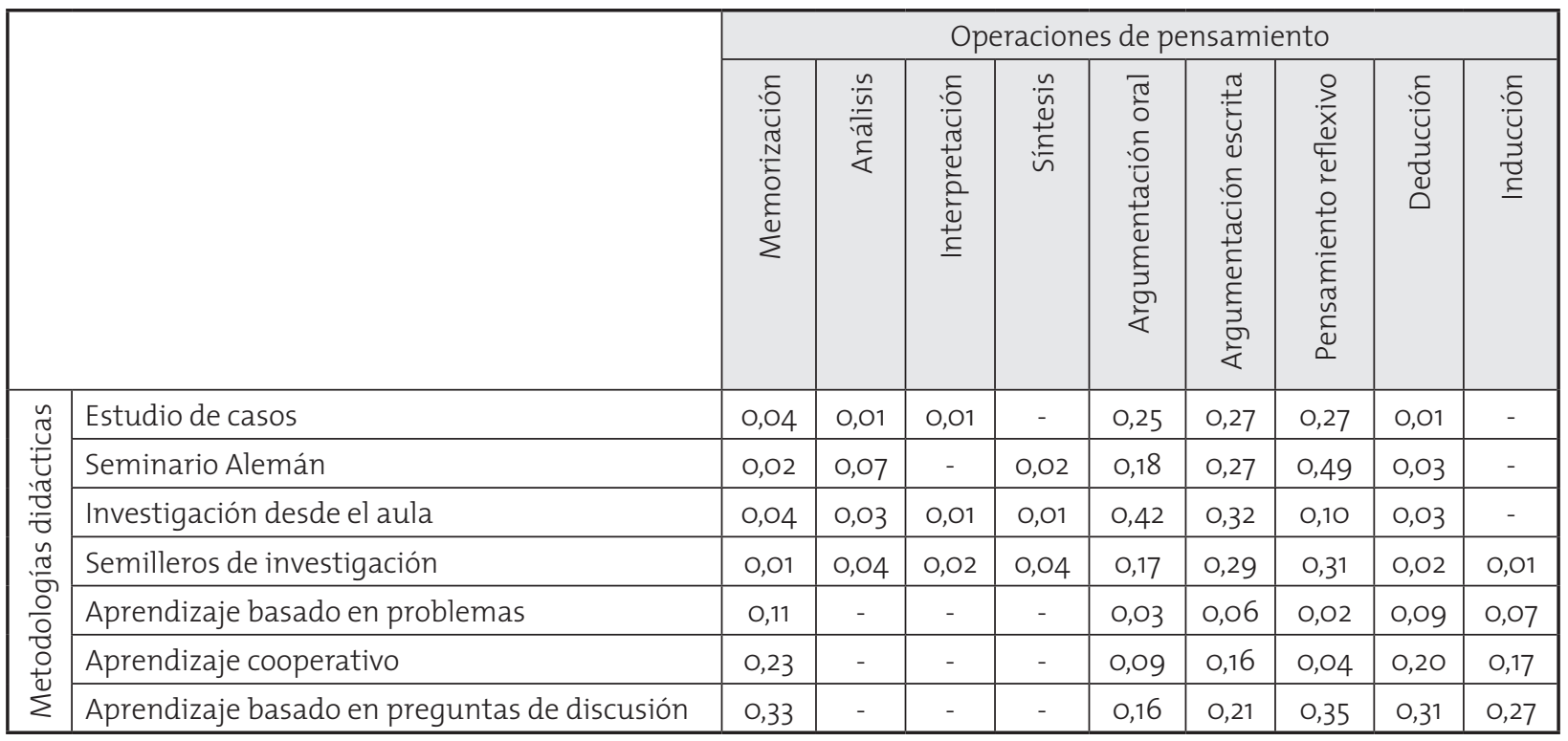

Fuente: resultados obtenidos con SPSS v22.

Pese a estos resultados, también sobresalen por la baja influencia las metodologías de aprendizaje cooperativo, las cuales presentan una relación del $23 \%$ con la memorización, $16 \%$ con la argumentación escrita y del $20 \%$ con la deducción. Esto se debe a que en la mayoría de los casos, los trabajos colaborativos giran alrededor de informes que resultan de consultas propuestas por los profesores, los cuales posteriormente son evaluados por medio de exposiciones de los estudiantes o exámenes escritos, explicándose el alto porcentaje en la memorización.

Esto contrasta con la afirmación de Bailey y Card (2009, p. 126) en el sentido de que el aprendizaje cooperativo les permite a los estudiantes la oportunidad de trabajar juntos para crear conocimiento y significado, en lugar de proporcionar datos e información para memorizar, lo cual, según las estadísticas, no se está cumpliendo en su totalidad. Sin embargo, también es claro que los profesores necesitan desarrollar habilidades en la aplicación de metodologías didácticas, de tal forma que se logre fortalecer la interpretación, el análisis y la sintesis en los estudiantes.

Además, las tecnologías de la información y la comunicación han llegado a formar parte importante en la aplicación de estrategias y metodologías didácticas como apoyo a los procesos de aprendizaje. La tabla 3 muestra la relación porcentual entre las metodologías didácticas y algunas herramientas tecnológicas de comunicación.

Se destacan el uso del correo electrónico y de las redes sociales como las herramientas de comunicación más utilizadas por los estudiantes para el desarrollo de sus actividades académicas. Por otro lado, los blogs, los wikis y los foros virtuales tienen muy poco uso a nivel de pregrado en las facultades de Administración. Algunos de los posibles factores de su bajo uso son: la poca infraestructura tecnológica de las instituciones de educación superior y la baja cultura tecnológica de los profesores. 
Tabla 3

Relación entre el uso de herramientas de comunicación y las metodologías didácticas

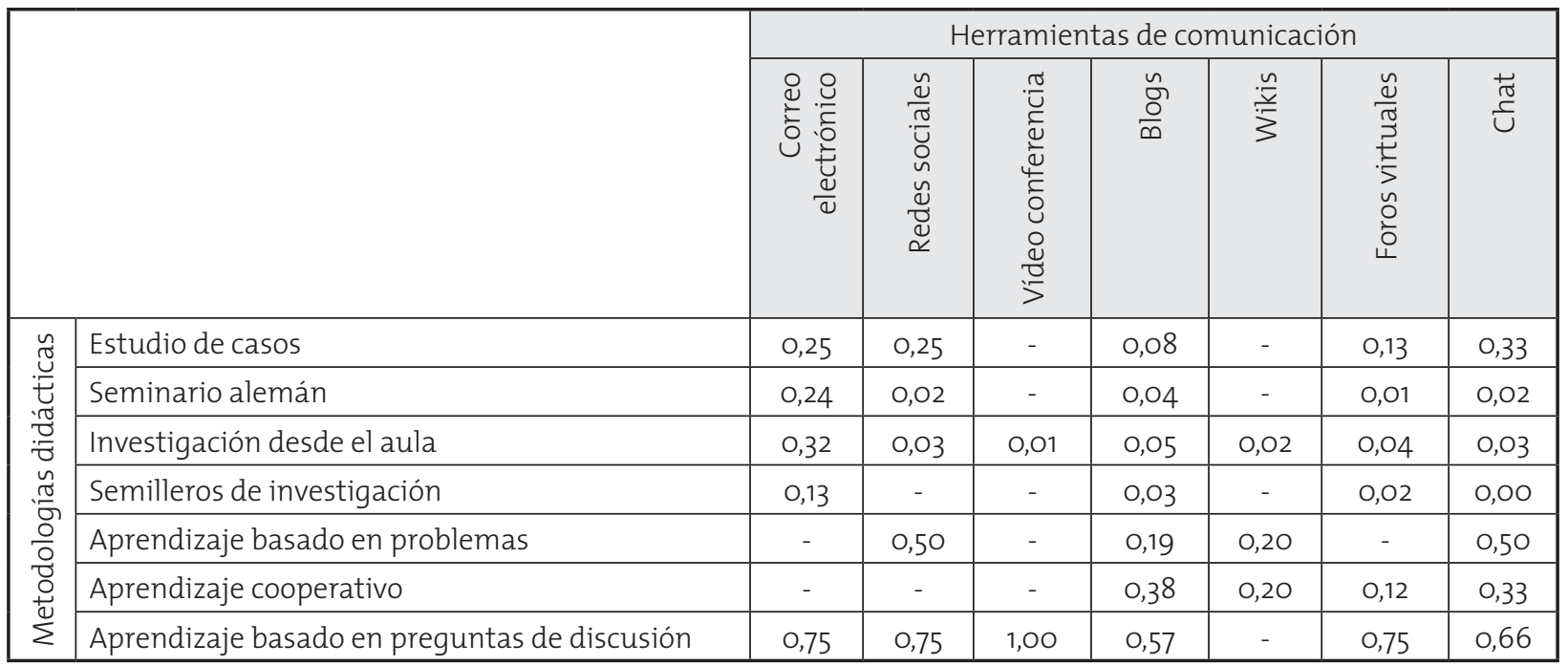

\section{Hallazgos obtenidos de la entrevista a los profesores y directivos de las facultades de Administración}

Con el fin de analizar la información obtenida a través de las entrevistas y los documentos, se realizó la siguiente clasificación de categorías de análisis con el fin de facilitar la identificación de comportamientos, las tendencias o los patrones en cada una de estas: planeación, conocimientos, competencias, enseñanza-aprendizaje, medios y recursos didácticos, y evaluación.

\section{Planeación}

Se evidenció que la planeación para llevar a cabo el proceso formativo se centraba en los contenidos o temas que se van a desarrollar; por tanto, hay un fuerte enfoque hacia la enseñanza y transmisión de contenidos. El 30\% de quienes explicaron su estrategia didáctica tienen en cuenta, además de los contenidos, las competencias por desarrollar, para luego poder definir los objetivos, los métodos didácticos, los recursos y las formas de evaluación; el $70 \%$ restante hace la planeación de los cursos fundamentados en la temática por desarrollar y las percepciones que tienen los profesores respecto a las necesidades de los estudiantes. Dentro de este último grupo, el $20 \%$ realiza evaluaciones diagnósticas para poder continuar con el proceso de planeación; además, los profesores buscan involucrar a sus pares para pensar los programas y hacer los ajustes respectivos.

Dentro de las observaciones hechas en esta primera fase de los procesos de aula, se recomienda socializar con los estudiantes los ejes temáticos que se van a seguir e involucrarlos en la discusión de las metodologías de enseñanza-aprendizaje, así como en las de evaluación, para que de esta manera se empiece a promover un aprendizaje significativo y aplicable para el desempeño de los futuros profesionales en su vida laboral.

Cabe agregar que en el proceso de planeación es necesario seguir una secuencia lógica para su desarrollo. El primer elemento para tener en cuenta es el diagnóstico en el entorno (institucional, regional y nacional), donde se evidencien las necesidades de formación de los estudiantes y las competencias por desarrollar, así como las habilidades de los 
profesores para el ejercicio de la docencia. Como segundo aspecto, identificar las temáticas, las metodologías y los recursos necesarios para el desarrollo de los cursos. Finalmente, es importante la socialización de las metodologías didácticas y de evaluación con los estudiantes.

\section{Los conocimientos}

No se cuenta con una cantidad suficiente para orientar tendencias de opiniones, pero se hacen conexiones interesantes entre lo importante que es para los estudiantes el desarrollo de sus capacidades y aprendizajes en medios laborales como prácticas profesionales, casos de estudio, mesas de debate, entre otros, con lo que se identificó como la formación por competencias, que es uno de los pilares fundamentales en las estrategias didácticas para obtener resultados positivos y medibles desde el punto de vista de los estudiantes, pues ellos podrán verificar en su propia vivencia cuáles son sus reacciones a situaciones de presión laboral o toma de decisiones, entre otras. La percepción de las intervenciones en este sentido da cuenta de la implementación no planificada de una estrategia didáctica que necesariamente se debe consolidar en un programa de formación por competencias con más contenidos y, de alguna manera, marca el camino que se debe recorrer.

Asimismo, se hace una tímida referencia a la precalificación de los estudiantes al entrar en cada uno de los pregrados, identificando esta como una herramienta para orientar las estrategias didácticas de cara a lo que el estudiante, desde su propio registro y matrícula, quiere aprender; es decir, se verifica su disposición para aprender y conocer determinado tema más que otros. Esta es una herramienta primordial a la hora de diseñar e implementar estrategias didácticas que, además, deberán ir acompañadas de instrumentos de medición de su efectividad, retroalimentación y corrección de fallas.

Con fundamento en las encuestas, se hace referencia a lo importante que es para el éxito de las estrategias didácticas y la formación por competencias la disposición que tenga el profesor; es decir, que las instituciones educativas deben realizar procesos de selección que se adecuen a las nuevas tendencias educativas y, con los profesores ya vinculados, se deben implementar programas y talleres en los cuales se les transmita la forma en que la universidad realizará sus procesos en el aula y aplicará sus estrategias didácticas, particularmente en el área del conocimiento. Se tendrán entonces que desarrollar desde las esferas jerárquicas altas metodologías de evaluación y retroalimentación de estas actividades, tanto en la vinculación de profesores como en la reorientación de los ya vinculados y que aún no estén alineados con las estrategias formuladas desde la dirección.

\section{Las competencias}

Los entrevistados resaltan la importancia que posee la inclusión de las competencias dentro del proceso de formación, donde sobresalen claramente algunas como: la toma de decisiones (60\%), la capacidad de análisis (20\%), el trabajo en equipo, así como el desarrollo de habilidades de lectura, redacción, ortografía e interpretación de textos, datos financieros y matemáticos; dentro de las competencias de lectura y escritura se destaca que estas contribuyen directamente a un manejo adecuado de las competencias comunicativas, las cuales son esenciales para todo tipo de interacciones sociales.

Los entrevistados identificaron las bases de la formación por competencias, haciendo alusión a los objetivos que se quieren lograr con esta metodología, pero sin muchos fundamentos. Son ideas que si bien están instintivamente orientadas a lograr los objetivos de la formación por competencias y la utilización de estrategias pedagógicas, no se cuenta con una estructura definida, una planeación de las actividades desarrolladas desde los objetivos y pasando por las estrategias para finalmente llegar a los programas y proyectos que deben enmarcar estas metodologías. En conclusión, se debe hacer un 
trabajo estructural desde la dirección de las universidades en este aspecto y firmar un pacto pedagógico con los profesores que contenga estos tópicos.

\section{Enseñanza-aprendizaje}

Sobre las metodologías didácticas que se aplican en las instituciones de educación superior en los programas de Administración por parte de los profesores, se lograron identificar las siguientes estadísticas:

\section{Figura 1}

\section{Porcentaje de aplicación de metodologías didácticas de acuerdo con el enfoque}

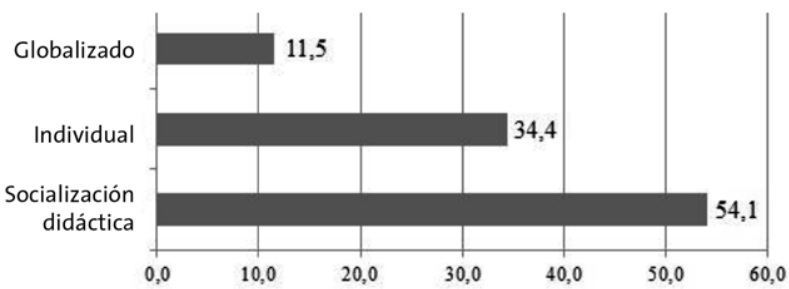

Fuente: elaboración propia a partir de los resultados obtenidos.
Entre las metodologías del enfoque de socialización didáctica, las que presentan mayor aplicación son: la clase magistral con un 21,3\% de desarrollo, trabajo en grupos heterogéneos y aprendizaje cooperativo con $11,5 \%$.

Por otra parte, dentro de las metodologías de enfoque individual, las que se presentaron con mayor aplicación son: método de investigación, con una participación del 18,1\%; sin embargo, cabe aclarar que el $8 \%$ de los profesores entrevistados definieron como método de investigación las consultas de material bibliográfico $\mathrm{El}$ otro método de mayor aplicación es el seminario alemán, con el 8,2\%.

Finalmente, el enfoque globalizado de metodologías didácticas presenta los siguientes porcentajes de uso: visita a empresas, $6,6 \%$, y juegos de empresa, 1,6\%. La figura 2 presenta un panorama de la aplicación de los métodos didácticos a partir de la información suministrada por los profesores entrevistados.

Figura 2

Porcentaje de aplicación de metodologías didácticas

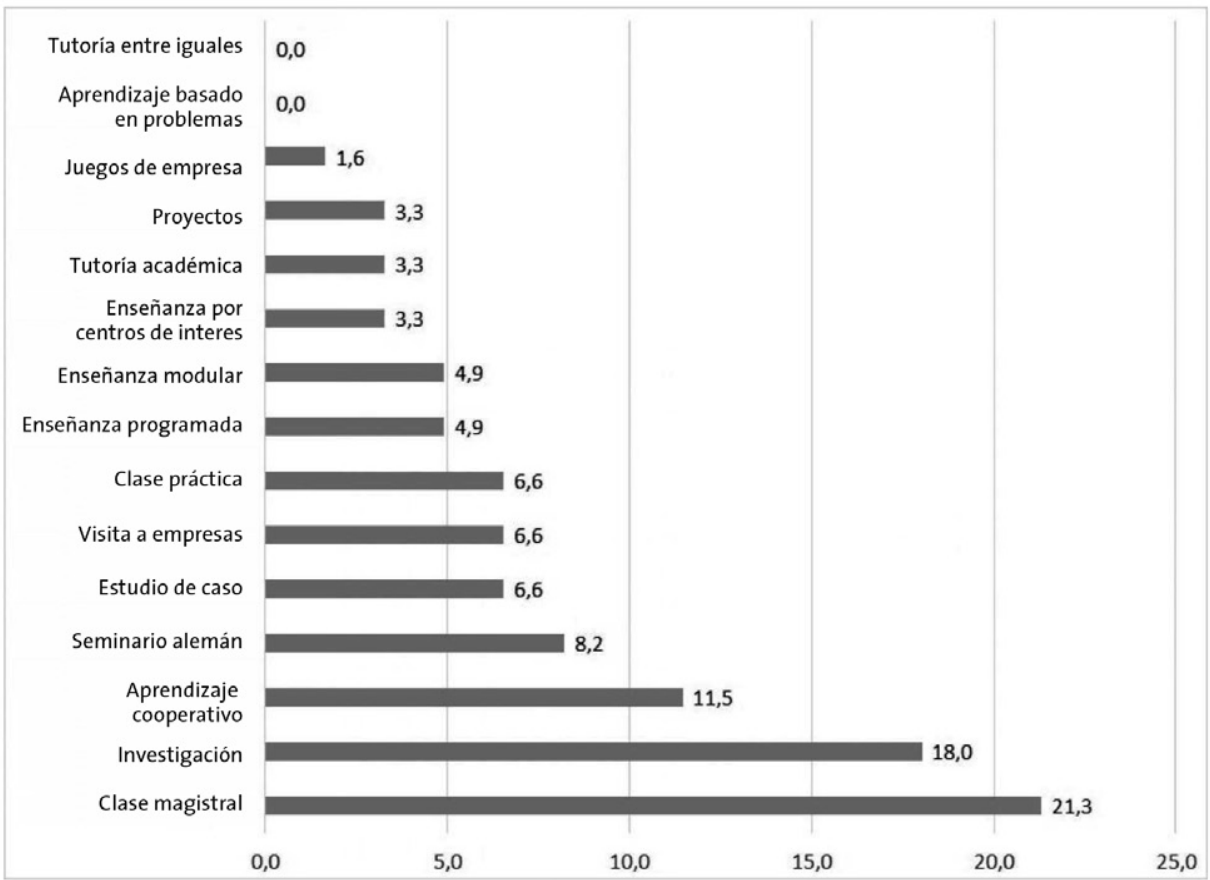




\section{Medios y recursos didácticos}

En las respuestas de los profesores se encontró que la virtualidad (35\%) tiene un potencial crecimiento, pero se advierte que, a pesar de su disponibilidad, no se le está dando el uso esperado debido a que muchos profesores se resisten a utilizar las nuevas tecnologías. Actualmente, las clases virtuales no están muy desarrolladas, pero se pueden usar las TIC para reforzar los procesos; puede verse que hay interés por incrementar su uso.

La metodología del caso es usada en un $22 \%$ para desarrollar las habilidades comunicativas de los alumnos y para ver cómo aplican la teoría a la práctica empresarial. Otra razón para su uso es que el caso es un medio que motiva e incentiva al alumno, porque al emplear casos actuales que se relacionen con la realidad empresarial, se les hace ver su utilidad.

Los talleres se referencian en un $13 \%$ como herramientas que permiten ejemplificar el escenario de una posible empresa, en la cual los alumnos pueden aplicar los conocimientos adquiridos. El taller le permite al estudiante interpretar un problema basado en su propio conocimiento. Su impacto en los primeros niveles posiblemente no sea tan significativo como sí lo puede ser en estudiantes de último nivel.

Los simuladores también tienen su relevancia, especialmente se menciona el simulador del juego de la bolsa de valores, donde por medio de diferentes escenarios se tiene en cuenta la variabilidad de indicadores con sus componentes micro y macroeconómicos para aprender a tomar decisiones, lo que permite el desarrollo de habilidades en los estudiantes.

La tecnología puede ser atractiva porque obliga a cuestionar la relación profesor-estudiante y, al mismo tiempo, obliga al docente a ir más allá de la simple transmisión de datos, y le exige preguntar y construir inquietudes con sus estudiantes.

Se observa que las TIC son una buena herramienta y que los profesores están de acuerdo con su utilización, pero al mismo tiempo dicen que no acceden a ellas. Hay profesores que afirman que "inicialmente esto representa una duplicación del trabajo de los cursos, pero que a la larga se ven los resultados". Se concluye, por tanto, que se debería tener más capacitación en el uso de estas herramientas.

En las universidades se ha ido capacitando también a los monitores de las materias que son los que apoyan al profesor en el conocimiento de estas metodologías; así, los monitores académicos ayudan a la construcción de esos programas con el apoyo de las nuevas tecnologías. Las universidades ahora tienen diferentes proyectos con el propósito de poner al servicio de los profesores plataformas para los recursos tecnológicos con el fin de apoyar su labor como profesor.

Según el marco teórico desarrollado y los resultados empíricos encontrados, los lineamientos estratégicos deben girar en función de lograr que los estudiantes se apropien de las TIC y construyan a partir de allí escenarios de diálogos temáticos, asesorados por los profesores, los cuales deben ser también proclives a estos cambios, a través del uso de blogs, chat, videoconferencias, aulas virtuales y aprendizajes autónomos guiados por las TIC.

\section{La evaluación}

A partir de las entrevistas realizadas a profesores, se hace una clasificación de los métodos de evaluación utilizados en el aula. La tabla 4 presenta estas estadísticas.

Las actividades que no suscitaron comentarios sobre su aplicación como método de evaluación fueron: los portafolios y el uso de diarios o blogs de curso.

Es de notar que el proceso de evaluación no se enfocaba en un solo método, sino en la combinación de varios. Entre los métodos de evaluación más destacados se encuentran los trabajos de consulta y los proyectos con actividades de desarrollo, los cuales, posteriormente, se socializan en los cursos usando 
Tabla 4

Porcentajes de aplicación de metodología de

\begin{tabular}{|c|c|}
\hline Metodología & 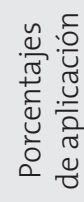 \\
\hline Trabajos y proyectos & 16 \\
\hline - Debate & \multirow{3}{*}{11} \\
\hline - Solución de casos & \\
\hline - Tareas reales o simulaciones & \\
\hline - Pruebas de desarrollo & \multirow{4}{*}{8} \\
\hline - Mapas conceptuales & \\
\hline - Pruebas orales & \\
\hline - Informe y memoria de prácticas & \\
\hline - Pruebas objetivas & \multirow{2}{*}{5} \\
\hline - Escala de actitudes & \\
\hline • Ensayos & \multirow{3}{*}{3} \\
\hline • Solución de problemas & \\
\hline - Observación: retroalimentación puntual & \\
\hline
\end{tabular}

Fuente: elaboración propia a partir de los resultados obtenidos.

métodos de evaluación oral, como: las exposiciones, los grupos de discusión o los debates.

Otra de las combinaciones de los métodos de evaluación que se destacan es la construcción de mapas conceptuales y pruebas objetivas o de desarrollo a partir de trabajos de consultas o del material desarrollado en clases magistrales.

Adicionalmente, el análisis y la solución de casos, junto con las tareas puntuales de prácticas en empresas o la simulación de situaciones, son vistos como actividades complementarias que evalúan no solo el dominio conceptual sino actitudes y habilidades específicas en los estudiantes, tales como el manejo de la información y la toma de decisiones. Sin embargo, los profesores comentan que aunque estos métodos resultan ser efectivos, su aplicación puede ser muy complicada cuando los cursos están conformados por un número muy grande de estudiantes.

\section{Conclusiones}

De acuerdo con los resultados obtenidos, se evidencia que las metodologías didácticas se enfocan cada vez más en la formación por competencias de los estudiantes. Sin embargo, esta tendencia sigue siendo tímida y, en algunos casos, no se encuentra alineada con los objetivos de formación, generando confusión en los estudiantes al no entender qué es lo que se busca con la aplicación de algunas metodologías como: las visitas a empresas, los estudios de casos, el seminario alemán, la investigación, el aprendizaje basado en problemas, entre otros.

Frente a esta tendencia a la formación por competencias, han surgido algunas críticas, las cuales tienen que ver con la función social de la universidad. Según Toro et al. (2013), la universidad se preocupa más por formar para la vida profesional y el trabajo, que para la discusión, la reflexión, la investigación y el pensar. En este orden de ideas, Gutiérrez y Villegas (2015) aseveran que en un contexto donde las reformas de políticas se basan en las necesidades del mundo empresarial y económico, se hace caso omiso de la función social de la universidad: el desarrollo intelectual de una sociedad.

Por otro lado, Brown et al. (2012) y Chesnut y Burley (2015) argumentan que las personas que no se dedican a una mejora sustantiva de las competencias, a través de cualquier aprendizaje formal o informal, corren cada vez más el riesgo de ser vulnerables en el mercado laboral, porque su capacidad de ser adaptables en cuanto a su evolución profesional puede decaer o corren cada vez más el riesgo de ser encerrados en formas particulares de trabajo.

En consecuencia, es necesario tener en cuenta ambos enfoques: el desarrollo intelectual y las habilidades para el trabajo. Sin embargo, con miras a una formación integral, se requiere no dejar de lado los aspectos éticos. Del mismo modo, se necesita que los directivos, profesores y estudiantes, como protagonistas del proceso de enseñanza-aprendizaje, busquen espacios de reflexión alrededor de la pertinencia y el uso de las estrategias y metodologías didácticas que 
ISSN $0123-1294$ | Educ.Educ. Vol. 19. No. 2 | Mayo-Agosto de 2016 | pp. 205-220.

Universidad de La Sabana | Facultad de Educación

contribuyan a la formación de personas éticas, con pensamiento crítico y habilidades para el trabajo.

Finalmente, en vista de que en la actual investigación no se abordó el tema de las metodologías didácticas y su relación con el desarrollo de las competencias necesarias en el contexto de la formación de un administrador, queda abierta la propuesta para futuras investigaciones.

\section{Referencias}

Alzate-Medina, G. M. y Peña-Borrero, L. B. (2010). La tutoría entre iguales: una modalidad para el desarrollo de la escritura. Peer Tutoring: Developing Writing in College Education, 9 (1), 123-138. Recuperado de www. scielo.org.co/pdf/rups/vgn1/vgnia1o.pdf

Amat, O. S. (1998). Aprender a enseñar una visión práctica de la formación de formadores (4 ed.). Barcelona: Ediciones Gestión 2000. Recuperado de http://www.iutep.tec.ve/uptp/images/Descargas/materialwr/ libros/OriolAmatSalas-AprenderAEnsenar.PDF

Baeten, M., Kyndt, E., Struyven, K. y Dochy, F. (2010). Using student-centred learning environments to stimulate deep approaches to learning: Factors encouraging or discouraging their effectiveness. Educational Research Review, 5 (3), 243-260.

Bailey, C. J.y Card, K. A. (2009). Effective pedagogical practices for online teaching: Perception of experienced instructors. Internet and Higher Education, 12 (3-4), 152-155.

Brown, A., Bimrose, J., Barnes, S.-A. y Hughes, D. (2012). The role of career adaptabilities for mid-career changers. Journal of Vocational Behavior, 80 (3), 754-761.

Burgess, J. R. D. y Russell, J. E. A. (2003). The effectiveness of distance learning initiatives in organizations. Journal of Vocational Behavior, 63: 289-303

Calderón, G. H., Castaño, G. D., Lozada, N. B., Gutiérrez, L. V., Pérez, P. H. y Posada, R. B. (2014). Generación de conocimiento en los grupos élite de investigación en administración en Colombia. Bogotá: Universidad Nacional de Colombia. Recuperado de http://www.libreriadelau.com/generacion-de-conocimiento-en-los-gruposelite-de-investigacion-en-administracion-en-colombia-investigacion-y-ciencia.html\#.VYwlFEarFno

Chesnut, S. R. y Burley, H. (2015). Self-efficacy as a predictor of commitment to the teaching profession: A meta-analysis. Educational Research Review, 15, 1-16.

Cid-Sabucedo, A., Pérez-Abellás, A. y Zabalza, M. A. (2009a). Las prácticas de enseñanza declaradas de los "Mejores Profesores" de la Universidad de Vigo. Relieve, 15 (2), 1-29. Recuperado de http://www.uv.es/RELIEVE/ V15n2/RELIEVEV15n2_7.htm

De Jesús, M. I., Méndez, R., Andrade, R. y Martínez, D. R. (2007). Didáctica: docencia y método. Una visión comparada entre la universidad tradicional y la multiversidad compleja. Revista de Teoría y Didáctica de las Ciencias Sociales, 1 (12), 9-29. 
De Miguel, M. D., Alfaro, I. J. R., Apodaca, P. U., Arias, J. M. B., García, E. J., Lobato, C. F. et al. (2005). Modalidades de enseñanza centradas en el desarrollo de competencias, orientaciones para promover el cambio metodológico en el espacio europeo de educación superior. Oviedo: Ediciones Universidad de Oviedo.

Fung, D. (2014). Promoting critical thinking through effective group work: A teaching intervention for Hong Kong primary school students. International Journal of Educational Research, 66, 45-62.

González, M. G. y Ramírez, I. R. (2010). Enseñar a aprender, un reto para la formación de profesionales universitarios en el nuevo siglo. ODICEO, Revista Electrónica de Pedagógía, 7 (14), 1. Recuperado de http://www. odiseo.com.mx/bitacora-educativa/ensenar-aprender-reto-para-formacion-profesionales-universitariosnuevo-siglo

Grisales-Franco, L. M. y González-Agudelo, E. M. (2009). El saber sábio el saber enseñado: un problema para didáctica universitária. Educación y Educadores, 12 (2), 77-86. Recuperado de http://dialnet.unirioja.es/ servlet/articulo?codigo $=3084419$

Gutiérrez, D. C. y Villegas, E. G. (2015). The importance of teaching methodology in higher education: A critical look. Procedia - Social and Behavioral Sciences, 174, 377-382.

Herrero, J. de J. (1996). Introducción a la enseñanza universitaria: didáctica para la formación del profesorado. Dykinson. Recuperado de https://books.google.com/books?id=G1GkAQAACAAJ\&pgis=1

Lile, R. y Kelemen, G. (2014). Results of researches on strategies of teaching/learning/sssessment based on interactive learning methods. Procedia - Social and Behavioral Sciences, 163, 120-124.

Lim, C. P.y Lee,J. C.-K. (2014). Teaching e-portfolios and the development of professional learning communities (PLCS) in higher education institutions. The Internet and Higher Education, 20, 57-59.

Lucio, A. R. (1989). Educación y pedagogía, enseñanza y didáctica: diferencias y relaciones. Revista de la Universidad de La Salle, 1 (17), 35-46.

Morales, P. y Landa, V. (2004). Aprendizaje basado en problemas. Theoria, Ciencia, Arte y Humanidades, 13 (1), 145-157. Recuperado de http://www.redaly c.org/resumen.oa?id=29901314

Nérici, I. G. (1985). Hacia una didáctica general dinámica. Kapelusz. Recuperado de https://books.google. com/books?id=eLHOPOAACAAJ\&pgis=1

Nikolova, I., van Ruysseveldt, J., De Witte, H. y Syroit, J. (2014). Work-based learning: Development and validation of a scale measuring the learning potential of the workplace (LPW). Journal of Vocational Behavior, $84(1), 1-10$.

Pedraza, N., Farías, G., Lavín, J.y Torres, A. (2013). Las competencias docentes en TIC en las áreas de negocios y contaduría. Un estudio exploratorio en la educación superior. Perfiles Educativos, 35 (139), 8-24.

Popil, I. (2011). Promotion of critical thinking by using case studies as teaching method. Nurse Education Today, 31 (2), 204-207. 
ISSN 0123-1294 | Educ.Educ. Vol. 19. No. 2 | Mayo-Agosto de 2016 | pp. 205-220.

Universidad de La Sabana | Facultad de Educación

Swanson, E. A., Nicholson, A. C., Boese, T. A., Cram, E., Stineman, A. M. y Tew, K. (2011). Comparison of selected teaching strategies incorporating simulation and student outcomes. Clinical Simulation in Nursing, 7 (3), e81-ego.

Tarabay, F. Y. (2009). Cualidades docentes universitarias: de la pedagogía a la relación afectivo-comunicativa. Revista de Teoría y Didáctica de las Ciencias Sociales, 1 (15), 355-377. Recuperado de http://www.redaly c. org/articulo.oa?id=65213215005

Tippelt, R. y Lindemann, H.-J. (2007). El método de proyectos. Recuperado de cmapspublic.ihmc.us/ rid=1KFJWWJ3B-11D27DY-1P5D/metodo proyectos.pdf

Toro, I. D. J., Roldán, J. I. R., Montenegro-Velandia, W., Hernández, B. Y. S., Vélez, C. P., Álvarez, M. J. et al. (2013). Educación y didáctica en el contexto del saber administrativo de los programas de administración: la formación del administrador.Xihmai, 8 (15), 7-34. Recuperado de http://dialnet.unirioja.es/servlet/articulo?codigo= 4164234\&info=resumen\&idioma=ENG

Verona, M. C. M. (2004). Métodos didácticos aplicables a materias de las disciplinas administrativas. De la lección magisterial al campus virtual. Tiempo de Educar, 5 (9), 89-114. Recuperado de http://www.redaly c. org/articulo.oa?id=31100904

Villamizar-Herrera, L. N., Montenegro-Velandia, W. y Salvador-Poveda, J. (2012). Revisión teórica sobre la enseñanza y aprendizaje de las matemáticas. Revista Virtual Universidad Católica del Norte, 1 (35), 254-287. Recuperado de http://revistavirtual.ucn.edu.co/index.php/RevistaUCN/article/view/361 